\title{
The Hartree-Fock triplet instability: influence of conformation and substitution
}

\author{
Christine Geron, George Dive *, Dominique Dehareng \\ Centre d'Ingéniérie des Protéines, Institut de Chimie B6, Sart Tilman, B4000 Liège 1, Belgium
}

Received 12 October 2005; received in revised form 17 November 2005; accepted 18 November 2005

\begin{abstract}
The triplet Hartree-Fock (HF) instability is investigated through the examples of substituted ethylenes and the small linear conjugated systems butadiene, hexatriene, octatetraene. A statistical analysis is performed for several conformations of the latters. The second eigenvalue of the instability matrix appears to be able to discriminate the groups of unsaturated compounds. The HF instability is largely influenced by conjugation and mesomeric effects and thus by the geometry. The number of $\pi$ electrons is also an important factor. The electronic correlation related to the HF instability is not quantified by the energy difference between the post-HF and HF levels.
\end{abstract}

(C) 2005 Elsevier B.V. All rights reserved.

Keywords: Hartree-Fock instabilities; Butadiene; Hexatriene; Octatetraene; Aminoethylene; Analysis of variance

\section{Introduction}

The Hartree-Fock (HF) wavefunction (wf) is a polyelectronic function based on three main concepts:

(i) The polyelectronic problem is solved in terms of products of monoelectronic contributions taking into account an average of the other electrons field [1]; thus, the simplest polyelectronic term is a Hartree product $\phi_{1}\left(\mathrm{c}_{1}\right) \phi_{2}\left(\mathrm{c}_{2}\right) \phi_{3}\left(\mathrm{c}_{3}\right) \ldots \phi_{\mathrm{N}}\left(\mathrm{c}_{\mathrm{N}}\right)$, the $\left\{\phi_{\mathrm{i}}\right\}$ being the monoelectronic functions, $c_{i}$ denoting the spatial and spin coordinates of electron $\mathrm{i}, \mathrm{N}$ being the number of electrons.

(ii) The HF wf is taken to be the Slater determinant $[2,3]$, hereafter noted D0, in order to obey the antisymmetry principle characterizing the fermions [4-6] or the Pauli exclusion principle $[7,8]$ for the electrons:

$\psi_{\mathrm{HF}}\left(c_{1}, c_{2}, \ldots, c_{\mathrm{N}}\right)=D 0=\left|\begin{array}{llll}\psi_{1}\left(c_{1}\right) & \psi_{2}\left(c_{1}\right) & \ldots & \psi_{\mathrm{N}}\left(c_{1}\right) \\ \psi_{1}\left(c_{2}\right) & \psi_{2}\left(c_{2}\right) & \ldots & \psi_{\mathrm{N}}\left(c_{2}\right) \\ \vdots & \vdots & \ldots & \vdots \\ \psi_{1}\left(c_{\mathrm{N}}\right) & \psi_{2}\left(c_{\mathrm{N}}\right) & \ldots & \psi_{\mathrm{N}}\left(c_{\mathrm{N}}\right)\end{array}\right|$

\footnotetext{
* Corresponding author. Tel.: +32 4 3663499; fax: + 3243663364 .

E-mail address: gdive@ulg.ac.be (G. Dive).
}

0166-1280/\$ - see front matter (C) 2005 Elsevier B.V. All rights reserved. doi:10.1016/j.theochem.2005.11.023
The monoelectronic functions $\psi_{\mathrm{k}}\left(c_{i}\right)$ are called spinorbitals. The spinorbitals are expressed as simple products of a spatial continuous function and a spin discrete function: $\psi_{i}(c) \Leftrightarrow \phi_{i}(\vec{r}) \sigma(s)$. (iii) the monoelectronic functions that are the elements of the Slater determinant are obtained in the framework of the variation calculus [9] with the restraint that the overlap integral matrix is given by $\left\langle\psi_{i} \mid \psi_{j}\right\rangle=\int \psi_{i}^{*}(c) \psi_{j}(c) \mathrm{d} c=\delta_{i j}$. This leads to the Fock equations $[5,6,10]:$

$F \psi_{i}=\sum_{j=1}^{N} \varepsilon_{i j} \psi_{j}$

$F$ being the Fock operator, depending on the solutions $\psi_{i}$ themselves. Any unitary transformation on the $\psi_{i}$ leaves the electronic density $\rho(\vec{r})$ unchanged and the particular and most largely used solutions leading to

$F \varphi_{i}=\varepsilon_{i} \varphi_{i}$

are called the canonical spinorbitals. They usually belong to an irreducible representation of the molecular system symmetry point group [11] and are thus delocalized.

On these general ideas, restraints can be imposed, leading to different Hartree-Fock (HF) frameworks.

a. All the $\sigma(s)$ are eigenfunctions of the monoelectronic spin operator $S_{z}$ (labelled $\alpha$ or $\beta$ ) and electrons are paired with the same $\phi_{i}(\vec{r})$ : this is the Restricted-HF framework (RHF). 
b. The electrons are all characterized by different $\phi_{i}(\vec{r})$ but still possess a spin function $\sigma(s)$ eigenfunction of the monoelectronic spin operator $S_{z}$ : this is the unrestricted-HF framework (UHF). If passing from a RHF to a UHF framework the energy decreases, this highights the existence of an external HF instability often called triplet instability.

c. The electrons are all characterized by different $\phi_{i}(\vec{r})$ and their spin functions $\sigma(s)$ are no longer eigenfunction of the monoelectronic spin operator $\mathrm{S}_{\mathrm{z}}$ : this is the generalized-HF framework (GHF). Passing from RHF or UHF to GHF with a decrease in energy is also associated with an external HF instability.

d. In the three a-c cases, the spatial function $\phi_{i}(\vec{r})$ can be optimized as real or complex and a decrease in energy, i.e. an instability, can also appear when going from real to complex.

e. In the three above a-c cases, the spatial function $\phi_{i}(\vec{r})$, called the molecular orbital (MO), belongs to an irreducible representation of the symmetry point group of the molecular system. This restraint can also be lifted inside a given HF framework, leading eventually to a so-called internal instability.

Hartree-Fock instabilities have been well known since the sixties essentially [12-45]. Slater as well as Coulson and Fisher $[12,13]$ were among the first to emphasize the existence of lower energy solutions related with relaxing restraints on the general framework presented above.

HF instabilities appear when a HF wavefunction obtained as a variational solution in one framework is no longer a minimum, or the best solution, in another framework. This is an artifact resulting from the simplicity of the monodeterminantal representation which becomes insufficient in specific cases. The particular case of the RHF- $>$ UHF instabilities is often referred to as triplet HF instabilities as first suggested by Cisek and Paldus [19]. It is already known [18-20,33,39,42,45] that all the conjugated unsaturated compounds are subject to this type of HF instability at their equilibrium geometry as well as compounds for which a diradicalar character appears $[32,34,40,42]$. In a previous study on a large set of molecules [42], it was emphasized that

(i) the localized character of the UHF wave function, expressing through the atomic spin densities (ASD), was due to its accounting for some kind of electronic correlation;

(ii) the larger this electronic correlation, the larger the HF instability;

(iii) the larger the resonance in cyclic polyenes, the lower the HF instability;

(iv) Heteroatoms influence triplet instabilities either through the geometry that they impose (boron, sulfur), or through their chemical nature or, more probably, through both features (oxygen, nitrogen).

The RHF wavefunction of symmetric cyclic polyenes or aromatic systems was found less unstable [45] than the one of linear conjugated systems thus highlighting the influence of the geometry and of the delocalization propensity of the $\pi$ cloud.

Many unanswered questions remain so far as for instance what properties of unsaturated compounds are responsible for the appearance of a triplet HF instability.

The aim of this work is to analyze the influence of the nature and the position of nitro and amino substituents of ethylene and to study the conjugation pattern incidence in polyenes on the observed triplet HF instability.

Section 2 is aimed at recalling the theoretical framework of the HF instabilities that will be dealt with in this article as well as the multivariate statistical basic concepts used in this work.

Section 3 presents the results and discussion about the substituted ethylenes.

Section 4 deals with the polyenes butadiene, hexatriene and octatetraene for, which a statistical analysis was performed.

Finally, a general conclusion is drawn in Section 5.

\section{Theoretical framework and statistical basic concepts}

Let the $\left\{\varphi_{\mathrm{k}}\right\}$ be the spinorbitals solutions of the variational problem in a specific HF framework. Some restraints are then lifted and the $\left\{\varphi_{\mathrm{k}}\right\}$ can be no longer the best functions leading to the local energy minimum in the new framework. Another reference determinant $D$ is constructed with new spinorbitals $\left\{\varphi_{\mathrm{k}}^{\prime}\right\}$ expanded on the first ones:

$\varphi_{\mathrm{k}}^{\prime}=\varphi_{\mathrm{k}}+\sum_{j=N+1}^{M} c_{j k} \varphi_{j}$

where $N$ is the number of occupied spinorbitals and $M$ is the total number of spinorbitals (occupied+virtuals). Thus, the new determinant takes the form:

$$
\begin{aligned}
D\left(\left\{\varphi_{k}^{\prime}\right\}\right)= & D 0\left(\left\{\varphi_{k}\right\}\right)+\sum_{i=1}^{N s} u_{i} D_{i}\left(\left\{\varphi_{k}\right\}\right)+\frac{1}{2} \\
& \times \sum_{i=1}^{N s} \sum_{j=1}^{N s} u_{i} u_{j} D_{i j}\left(\left\{\varphi_{k}\right\}\right)+\ldots
\end{aligned}
$$

where the index (indices) $i(i, j) \operatorname{stand}(s)$ for the replacements of occupied spinorbital $(s) k(k, l)$ in $D 0$ by the virtual(s)a $(a, b)$ in $D_{i}\left(D_{i j}\right): i \equiv k \rightarrow a, j \equiv l \rightarrow b$. The coefficients $u_{i}$ are equal to the $c_{j k}$. The change in the expectation value $E=<D|H| D>/<$ $D \mid D>$ related with the hamiltonian $H$ for a change of $D$ in the vicinity of $D 0$ is considered up to the second order. Keeping in mind that $D 0$ is a variational solution, this leads to

$$
\begin{aligned}
\Delta E & \approx \sum_{i=1}^{N s} \sum_{j=1}^{N s}\left[u_{i}^{*} u_{j} A_{i j}+\frac{1}{2}\left(u_{i}^{*} u_{j}^{*} B_{i j}+u_{i} u_{j} B_{i j}^{*}\right)\right] \\
& \approx \frac{1}{2}\left(\begin{array}{ll}
U & U^{*}
\end{array}\right)\left(\begin{array}{cc}
A & B \\
B^{*} & A^{*}
\end{array}\right)\left(\begin{array}{c}
U \\
U^{*}
\end{array}\right) \\
& =\frac{1}{2}\left(\begin{array}{ll}
U & U^{*}
\end{array}\right) \mathcal{H}\left(\begin{array}{c}
U \\
U^{*}
\end{array}\right)
\end{aligned}
$$

with an asterisk exponent denoting the complex conjugate. 
Let us point out here that the $A$ matrix is a single excitation configuration interaction matrix while the $B$ matrix only implies double excitations of the reference determinant.

If the $i$ and $j$ indices represent single excitations respectively from $\varphi_{\mathrm{k}}(c)$ (occupied spinorbital) to $\varphi_{\mathrm{a}}(c)$ (virtual spinorbital) and from $\varphi_{1}(c)$ (occupied) to $\varphi_{\mathrm{b}}(c)$ (virtual), the $\mathrm{A}$ and $\mathrm{B}$ elements write:

$\mathbf{A}_{i j}=\left(\varepsilon_{a}-\varepsilon_{k}\right) \delta_{i j}+<a k|l b>-<a b| l k>$

$\mathbf{B}_{i j}=<a k|l b>-<a l| b k>$

$<a b \mid k l>=\iint \varphi_{a}(\mathrm{c} 1) \varphi_{b}(\mathrm{c} 1) \frac{1}{r_{12}} \varphi_{k}(\mathrm{c} 2) \varphi_{l}(\mathrm{c} 2) \mathrm{dc} 1 \mathrm{dc} 2$

If the determinant $D 0$ is stable, any displacement away from its spinorbitals will be characterized by an increase of energy: $\Delta E$ is positive. Otherwise, $\Delta E$ is negative. The hermitian quadratic form must then be positive definite if $D 0$ is to be a stable minimum, i.e. the eigenvalues $\lambda$ of the characteristic equation

$$
\left(\begin{array}{cc}
A & B \\
B^{*} & A^{*}
\end{array}\right)\left(\begin{array}{c}
U \\
U^{*}
\end{array}\right)=\mathscr{H}\left(\begin{array}{c}
U \\
U^{*}
\end{array}\right)=\lambda\left(\begin{array}{c}
U \\
U^{*}
\end{array}\right)
$$

are required to be positive.

Eq. (5) are the conditions for the stability of the HF wavefunction expressed as $D 0$ and have been derived and presented by many authors in the early litterature [see for instance the pioneering works [14-31].

The instabilities that are treated in this work are characterized by a RHF initial determinant $D 0$ in Eq. (2) and a UHF new determinant $D$. They are referred to in the litterature as triplet instabilities.

The eigenvectors $U$ of $\mathscr{H}$ provide the weight of the excitations $i \equiv k \rightarrow a$ in the new determinant and the nature of the MO $(k, a)$ involved.

The results were obtained using the program GaUsSIAN98 [46] using a 6-311G** basis set $[47,48]$. The geometries were optimized at the RHF level and further calculations were also performed at the correlated MP2 [49] or QCISD [50] levels or at the density functional B3LYP [51] level.
In statistical analysis [52], the variance/covariance, or simply covariance, and the correlation matrices are key features. Covariance and correlation are related parameters that indicate the extent to which two random variables co-vary. Given any pair of random variables $X_{i}$ and $X_{j}$, for which $\mathrm{K}$ measures were conducted, their covariance is defined as

$\operatorname{cov}\left(X_{i}, X_{j}\right)=\sum_{k=1}^{K}\left(X_{k, i}-\bar{X}_{i}\right)\left(X_{k, j}-\bar{X}_{j}\right) /(K-1)$

where $\bar{X}_{i}$ denotes the means of $X_{i}$. The covariance of a variable with itself is called the variance, $\operatorname{cov}\left(X_{i}, X_{i}\right)=\operatorname{var}\left(X_{i}\right)$ the square root of which is the standard deviation $\sigma_{i}$. The correlation $\rho_{i j}$ of two random variables $X_{i}$ and $X_{j}$ is defined as

$\rho_{i j}=\operatorname{cov}\left(X_{i}, X_{j}\right) / \sigma_{i} \sigma_{j}$

For a set of $n$ variables, the matrices whose elements are the covariances or the correlations are called covariance and correlation matrices. They are the basis of many statistical analyses, the most obvious consisting in testing the degree of independency of the variables, starting from the so-called null hypothesis $H_{0}$ implying that all the variables are completely independant, i.e. $\rho_{i j}=0(i \neq j)$. The test is run through the evaluation of the parameters $t_{i j}$ given by

$t_{i j}=\rho_{i j} \sqrt{K-2} / \sqrt{1-\rho_{i j}^{2}}$

that follow the Student's distribution with $K-2$ degrees of freedom. The tables for the error tests concerning the Student's distribution give the level of confidence $\left(C_{1}\right.$ in the Tables) that the calculated $t_{i j}$ fit in with the null hypothesis, i.e. $t_{i j}=0$. The lower the level of confidence, the less adequate the null hypothesis or the higher the interdependency beween the variables.

The canonical variance analysis is a sorting procedure based on an a priori definition of groups resulting in a pooled covariance-within-groups matrix $\mathbf{W}$ calculated as follows:

$W_{s t}=\sum_{i=1}^{N g} \sum_{k=1}^{N i}\left(X_{k i, s}-\bar{X}_{i, s}\right)\left(X_{k i, t}-\bar{X}_{i, t}\right) /(K-1)$

Table 1

C-C bond lengths $(\AA)$ and smallest eigenvalue of $\mathcal{H}, \lambda 1$, for substituted ethylene critical point (cp) geometries in their symmetry point group PG

\begin{tabular}{|c|c|c|c|c|c|c|}
\hline Species PG (cp) & $\mathrm{C}-\mathrm{C}$ & $\lambda 1$ (a.u.) & Mos $\mathrm{k} \rightarrow \mathrm{a}$ & $U_{i}$ & $p_{y}(\mathrm{~N} 3,4) \mathrm{MO}(\mathrm{k})$ & $p_{y}(\mathrm{~N} 3,4) \mathrm{MO}(\mathrm{a})$ \\
\hline Ethylene $D_{2 \mathrm{~h}}(\mathrm{~min})$ & 1.3163 & 0.00738 & $\mathrm{H} \rightarrow \mathrm{L}$ & 0.6751 & & \\
\hline Nitro-ethylene $\mathrm{C}_{\mathrm{s}}(\mathrm{min})$ & 1.3101 & -0.03545 & $\mathrm{H}-1 \rightarrow \mathrm{L}$ & 0.5687 & 0.0026 & 0.2297 \\
\hline Nitroso-ethylene $\mathrm{C}_{1}(\mathrm{~min})$ & 1.3163 & -0.03921 & $\mathrm{H}-1 \rightarrow \mathrm{L}$ & 0.4745 & 0.0287 & 0.2401 \\
\hline Amino-ethylene $C_{1}(\mathrm{~min})$ & 1.3217 & 0.01973 & $\mathrm{H} \rightarrow \mathrm{L}+3$ & 0.6128 & 0.2238 & 0.1822 \\
\hline Amino-ethylene $\mathrm{C}_{\mathrm{s}}(\mathrm{TS})$ & 1.3250 & 0.02329 & $\mathrm{H} \rightarrow \mathrm{L}+3$ & 0.6470 & 0.2505 & 0.1956 \\
\hline $1,1^{\prime}$-diamino-ethylene $\mathrm{C}_{2 \mathrm{v}}(\mathrm{PC} 3)$ & 1.3378 & 0.05790 & $\mathrm{H} \rightarrow \mathrm{L}+5$ & 0.6589 & 0.1655 & 0.1941 \\
\hline 1,1'-diamino-ethylene $\mathrm{C}_{\mathrm{s}}(\mathrm{TS})$ & 1.3310 & 0.04255 & $\mathrm{H} \rightarrow \mathrm{L}+4$ & 0.5871 & 0.1506 & 0.1531 \\
\hline 1,1'-diamino-ethylene $\mathrm{C}_{2}(\mathrm{~min})$ & 1.3288 & 0.04046 & $\mathrm{H} \rightarrow \mathrm{L}+4$ & 0.4913 & 0.1373 & 0.1511 \\
\hline trans-diamino-ethylene $\mathrm{C}_{2 \mathrm{~h}}(\mathrm{PC} 4)$ & 1.3210 & 0.01007 & $\mathrm{H} \rightarrow \mathrm{L}+5$ & 0.6375 & 0.2312 & 0.1718 \\
\hline trans-diamino-ethylene $\mathrm{C}_{2}(\mathrm{~min})$ & 1.3191 & 0.01403 & $\mathrm{H} \rightarrow \mathrm{L}+3$ & 0.6248 & 0.1827 & 0.1380 \\
\hline cis-diamino-ethylene $\mathrm{C}_{\mathrm{s}}(\mathrm{TS})$ & 1.3208 & 0.01838 & $\mathrm{H} \rightarrow \mathrm{L}+3$ & 0.6030 & 0.1907 & 0.1391 \\
\hline cis-diamino-ethylene $\mathrm{C}_{2}(\mathrm{TS})$ & 1.3193 & 0.01361 & $\mathrm{H} \rightarrow \mathrm{L}+3$ & 0.6036 & 0.1843 & 0.1330 \\
\hline
\end{tabular}

The highest component in the eigenvector is characterized through the value of the element $U_{i}$ and the nature of the MOs involved (occupied $k$ replaced by virtual a). $H$ and $L$ designate respectively the HOMO and LUMO. The LCAO coefficients on the $p_{y}$ basis function on the nitrogen(s) in MO $k$ and $a$ are also given. 


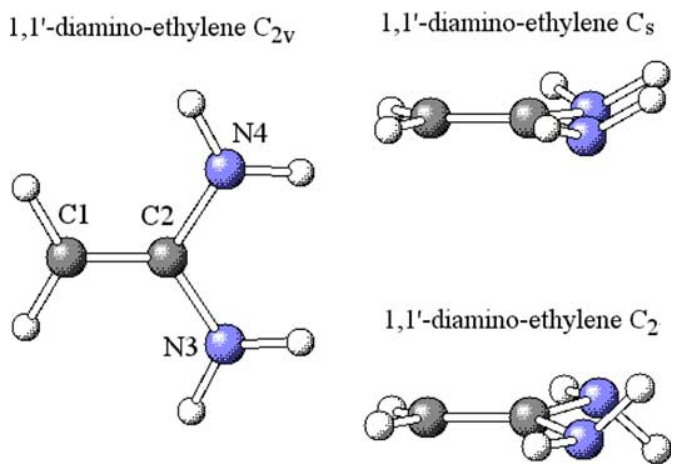

Scheme 1.

where $N_{g}$ is the number of chosen groups, $N_{i}$ the number of observations in the $i$ th group, $\bar{X}_{i, s}$ is the means of $X_{s}$ in the $i$ th group, and $K=\sum_{i=1}^{N g} N_{i}$. If the usual covariance matrix involving the whole set of variables (Eq. (6)) is noted $T$, a third matrix

$\mathbf{B}=\mathbf{T}-\mathbf{W}$

is easily calculated that is referred to as the covariancebetween-groups. The variable that best discriminates between the groups is the combination of the initial variables $\mathrm{C}^{\prime} \mathrm{X}$ that maximizes the covariance-between-groups while minimizing the covariance-within-groups. This is written as an eigenproblem solving:

$\left(\mathbf{W}^{-1} \mathbf{B}-\boldsymbol{\omega} \mathbf{I}\right) \mathbf{C}=0$

The statistical results were obtained with the program SAS [53].

\section{Substituted ethylene}

Amino and nitro substituents were considered as presented in Table 1. The 1,1'-diaminoethylene in conformations $\mathrm{C}_{2 \mathrm{v}}, \mathrm{C}_{\mathrm{s}}$ and $\mathrm{C}_{2}$ is presented in Scheme 1. These are respectively a critical point of index 3 (PC3), a transition state (TS) and a minimum, characterized by three, one and zero imaginary frequencies. The smallest eigenvalue of $\mathscr{H}$ (Eq. (5)), noted $\lambda 1$, and the main component in its related eigenvector are considered. The latter is analyzed in terms of its element $U_{i}$ value (Eq. (5)) and of the mean value of the LCAO coefficients on the nitrogens $p_{y}$ basis functions for the occupied $\rightarrow$ virtual MOs involved. These are always $\pi \rightarrow \pi^{*}$ excitations for planar systems or $\left(\pi \rightarrow \pi^{*}\right)$-like excitations for non planar systems.

Comparing monosubstituted compounds, the influence of $\mathrm{NH}_{2}$ or $\mathrm{NO}_{\mathrm{x}}$ is very different since the first produces an increase in $\lambda 1$ while the second produces a decrease leading yet to a negative value. From the analysis of the LCAO coefficients, the nitrogen $(\mathrm{s}) p_{\mathrm{y}}(\pi)$ basis functions are populated similarly in the occupied and virtual MOs involved in the instability eigenvector while this is not the case for the nitro compounds. For the latter, the occupied MO has a large population on the oxygens (not shown), but not on the nitrogen while the virtual has. As to the $U_{i}$ elements, no relation seems to emerge: they are of the order of 0.6 for most of the amino
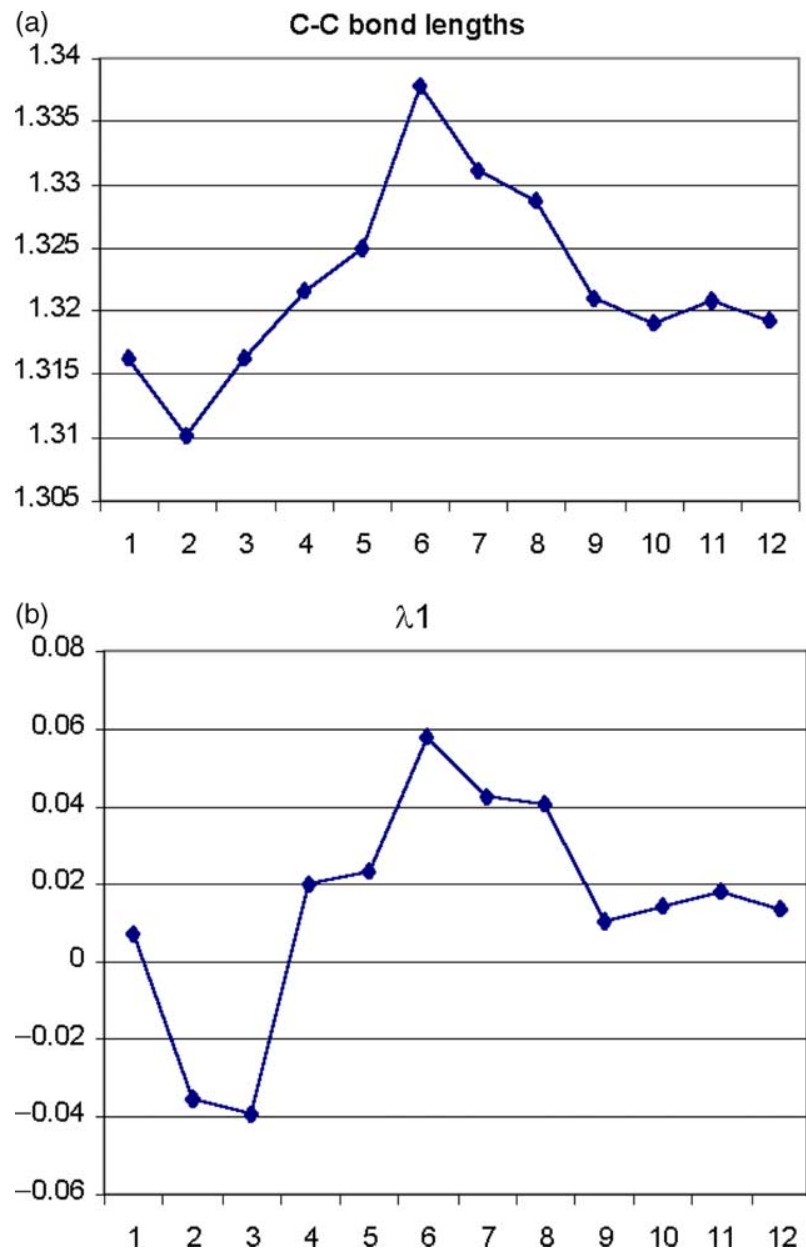

Fig. 1. C-C bond lengths ( $\AA$ ) and smallest eigenvalue of $\mathscr{H}, \lambda 1$ (a.u.), for the substituted ethylene compounds in the order given by Table 1 .

compounds except for the 1-1'-diamino $\mathrm{C}_{2}$ end $\mathrm{C}_{\mathrm{s}}$ which have comparable elements as the nitro and nitroso.

For all the compounds except the trans-diamino-ethylene, the planarity of the molecule favors an increase in $\lambda 1$, leading to suggest that the conjugation would have a role to play. The exception of the trans case is an enigma.

As to the relative position of the disubtitutions, the $1-1^{\prime}$ diamino and the trans or cis-diamino isomers present reverse

Table 2

$\mathrm{C}-\mathrm{C}$ bond length $(\AA)$, lowest eigenvalue of $\mathcal{H}, \lambda 1$ (a.u.) and absolute value of the atomic spin density (ASD) on each C for the benzene in the $D_{6 \mathrm{~h}}$ symmetry point group

\begin{tabular}{lll}
\hline $\mathrm{CC}$ & $\lambda 1$ & $\operatorname{ASD}(\mathrm{C})$ \\
\hline 1.485366 & -0.0644 & 0.156 \\
1.435366 & -0.0440 & 0.120 \\
1.385366 & -0.0225 & 0.080 \\
1.365366 & -0.0135 & 0.061 \\
1.345366 & -0.0042 & 0.033 \\
1.335366 & 0.0005 & 0.0 \\
1.325366 & 0.0052 & 0.0 \\
1.305366 & 0.0149 & 0.0 \\
1.285366 & 0.0249 & 0.0 \\
\hline
\end{tabular}




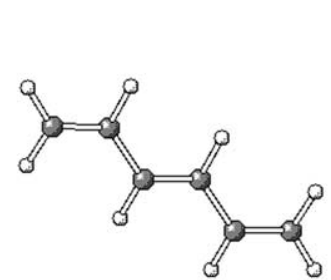

Hexatriene

Min 180.0180 .0

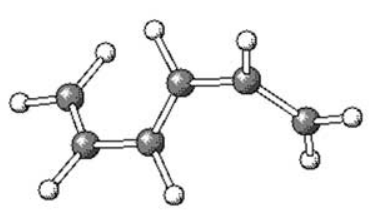

Min 39.039 .0

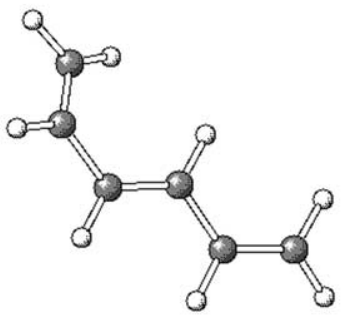

Min 37.0180 .0

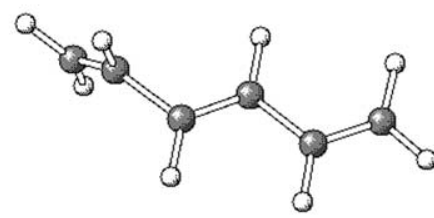

TS 102.080 .0

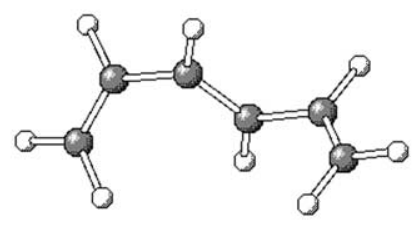

TS 41.0103 .0

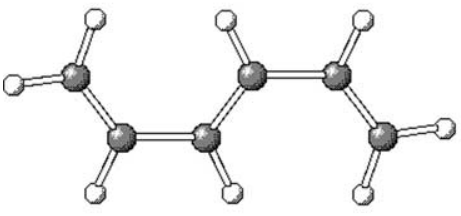

PC2 0.00 .0<smiles>O=C(O)OC(=O)OC(=O)OC(=O)OO</smiles>

PC2 102.0102 .0

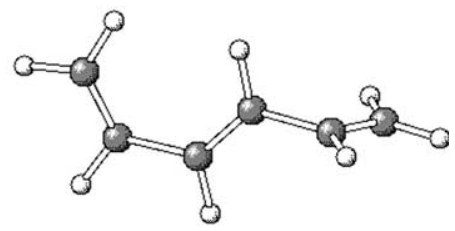

PC2 102.00 .0

Fig. 2. Optimized conformations of the hexatriene, showing the nature of the critical points and the values of the two $\mathrm{C}=\mathrm{C}-\mathrm{C}=\mathrm{C}$ dihedrals (see $\mathrm{Table} 4$ ).

\section{Octatetraene}

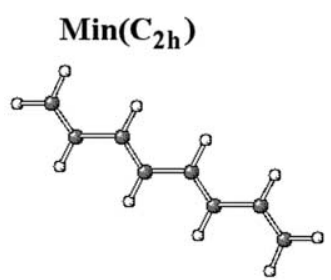

$\operatorname{TS}\left(\mathrm{C}_{2}\right)$

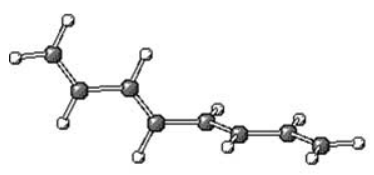

$\operatorname{Min}\left(\mathrm{C}_{2}\right)$<smiles>O=C(O)OC(=O)OC(=O)OC(=O)OC(=O)OC(=O)O</smiles>

$\operatorname{PC} 3\left(\mathrm{C}_{2 \mathrm{v}}\right)$



$\operatorname{TS}\left(\mathrm{C}_{2 \mathrm{v}}\right)$<smiles>O=C(O)C(=O)OC(=O)C(=O)OC(=O)C(=O)O</smiles>

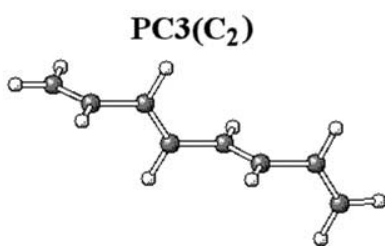

Fig. 3. Optimized conformations of the octatetraene, showing the nature of the critical points (see Table 5).

characteristics by reference to the monoamino ethylene: the former have larger $\lambda 1$ while the latter have smaller $\lambda 1$. All these observations can be put in relation with the $\mathrm{C}=\mathrm{C}$ bond lengths, as sketched in Fig. 1. The instability was previously related with a radicalar character of the wave function as given by the atomic spin density obtained after reoptimization (ASD) $[42,45]$. Few calculations on benzene emphasize that the instability increases ( $\lambda 1$ becomes more negative) when the

Table 3

The three lowest eigenvalues of $\mathcal{H}, \lambda 1-\lambda 3$, in a.u., for four conformations of the butadiene characterized as criticla points (cp)

\begin{tabular}{lccccc}
\hline Conformation (cp) & $\phi$ & $\Delta \mathrm{E}_{\text {sta }}$ & $\lambda 1$ & $\lambda 2$ & $\lambda 3$ \\
\hline Trans-butadiene (min) & 180.0 & -4.891 & -0.02304 & 0.04760 & 0.25213 \\
Cis-butadiene (min) & 40.31 & -1.661 & -0.01293 & 0.03088 & 0.24485 \\
Planar-cis-butadiene (TS) & 0.0 & -7.050 & 0.02746 & 0.03957 & 0.22620 \\
twis-butadiene (TS) & 100.46 & 0.0 & 0.00258 & 0.01111 & 0.26369 \\
\hline
\end{tabular}

The dihedral $\phi=\mathrm{C}=\mathrm{C}-\mathrm{C}=\mathrm{C}$ is given in degrees, as well as the stabilisation energy $\Delta E_{\text {sta }}$ in $\mathrm{kJ} / \mathrm{mol}$. 
Table 4

The three lowest eigenvalues of $\mathscr{H}, \lambda 1-\lambda 3$, in a.u., for eight conformations of the hexatriene

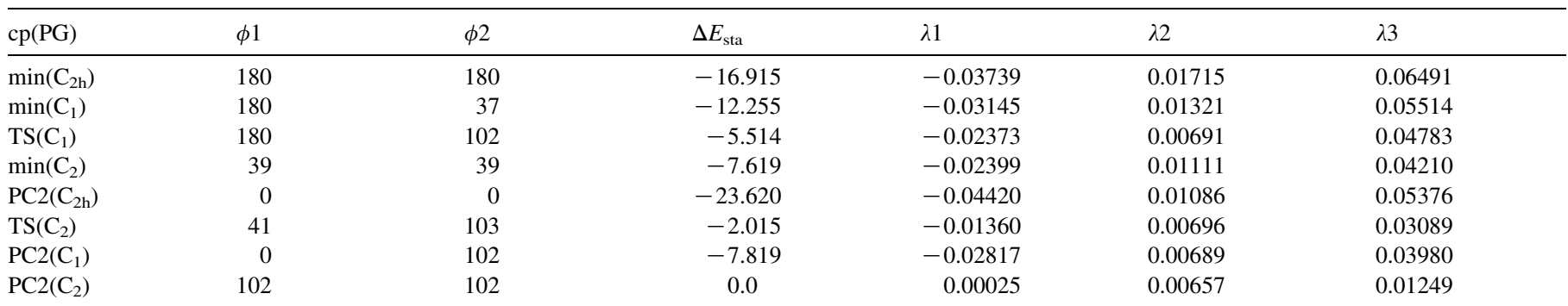

The conformations are characterized as critical points (cp) from the Fig. 2, with their symmetry point group PG. The dihedrals $\phi i$ are given in degrees and the stabilisation energy $\Delta E_{\text {sta }}$ in $\mathrm{kJ} / \mathrm{mol}$.

Table 5

The four lowest eigenvalues of $\mathscr{H}, \lambda 1-\lambda 4$, in a.u., for six conformations of the octatetraene

\begin{tabular}{|c|c|c|c|c|c|c|c|c|}
\hline $\mathrm{cp}(\mathrm{PG})$ & $\phi 1$ & $\phi 2$ & $\phi 3$ & $\Delta E_{\text {sta }}$ & $\lambda 1$ & $\lambda 2$ & $\lambda 3$ & $\lambda 4$ \\
\hline $\min \left(\mathrm{C}_{2 \mathrm{~h}}\right)$ & 180 & 180 & 180 & -31.310 & -0.04599 & -0.00206 & 0.04158 & 0.07279 \\
\hline $\min \left(C_{2}\right)$ & 180 & 31 & 180 & -25.725 & -0.04033 & -0.00776 & 0.04199 & 0.06324 \\
\hline $\mathrm{TS}\left(\mathrm{C}_{2 \mathrm{v}}\right)$ & 180 & 0 & 180 & -34.843 & -0.04862 & -0.00435 & 0.04025 & 0.06855 \\
\hline $\operatorname{PC} 3\left(\mathrm{C}_{2 \mathrm{v}}\right)$ & 0 & 0 & 0 & -42.256 & -0.05343 & -0.00948 & 0.03182 & 0.06139 \\
\hline $\mathrm{PC} 3\left(\mathrm{C}_{2}\right)$ & 100 & 100 & 100 & -0.016 & -0.00098 & 0.00359 & 0.00899 & 0.01326 \\
\hline
\end{tabular}

The conformations are characterized as critical points (cp) from the Fig. 3, with their symmetry point group PG. The dihedrals $\phi$ i are given in degrees and the stabilisation energy $\Delta E_{\text {sta }}$ in $\mathrm{kJ} / \mathrm{mol}$.

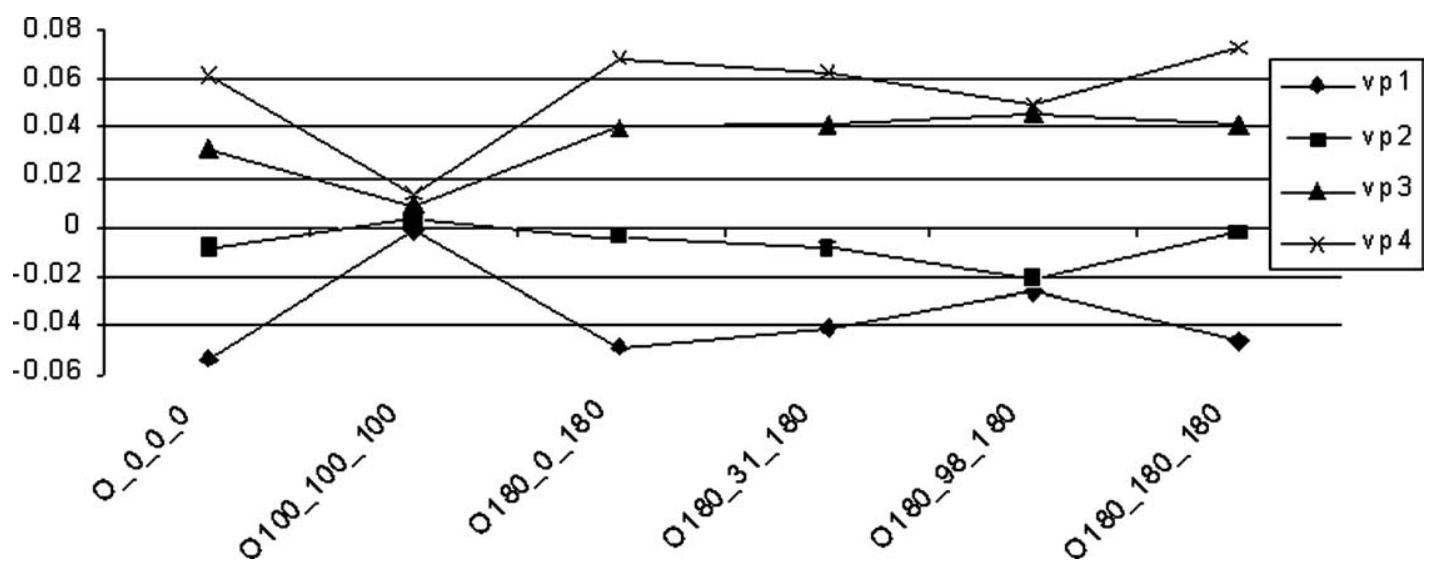

Fig. 4. Graph of the four lowest eigenvalues of $\mathcal{H}, \lambda i$, for the octatetraene conformations. The abscissas of the graph are labelled by the initial of the unsaturated compound followed by the value(s) of the dihedrals in the corresponding conformer.

distance CC increases, with a parallel increase in the ASD of the reoptimized UHF wave function (Table 2). This variation is similar to what is observed along the dissociation curve of any diatomic system, $\mathrm{H}_{2}$ being a very well-known textbook example, though the nature of the bonds involved are different ( $\pi($ benzene $\left.) / / \sigma\left(\mathrm{H}_{2}\right)\right)$. This is due to the appearance of some radicalar character when pulling partners apart. In the amino and diamino-ethylenes, the opposite is observed: now the stability increases as the $\mathrm{CC}$ bond length increases. This could be explained by the amino substituent trend to delocalize the $\pi$ electronic system thus counteracting any tendency for these electrons to localize on their site. In such delocalized systems, the electronic correlation should be smaller since a relationship
Table 6

Instability-related quantities $\lambda i / n_{\pi}$ (a.u.) and $\Delta E_{\text {sta }} / n_{\pi}(\mathrm{kJ} / \mathrm{mol})$ for nine of the studied conformers

\begin{tabular}{llrl}
\hline Compound & $\lambda 1 / n_{\pi}$ & \multicolumn{1}{c}{$\lambda 2 / n_{\pi}$} & $\Delta E_{\text {sta }} / n_{\pi}$ \\
\hline trans-butadiene & -0.00576 & 0.01190 & -1.2237 \\
$\min \left(\mathrm{C}_{2 \mathrm{~h}}\right)$ hexatriene & -0.00623 & 0.00286 & -2.8193 \\
$\min \left(\mathrm{C}_{2 \mathrm{~h}}\right)$ octatetraene & -0.00575 & -0.00026 & -3.914 \\
cis-butadiene & -0.00323 & 0.00772 & -0.4152 \\
$\min \left(\mathrm{C}_{2}\right)$ hexatriene & -0.00400 & 0.00185 & -1.2698 \\
$\min \left(\mathrm{C}_{2}\right)$ octatetraene & -0.00504 & -0.00097 & -3.2150 \\
planar-cis-butadiene & -0.00686 & 0.00989 & -1.7625 \\
PC2 $\left(\mathrm{C}_{2 \mathrm{~h}}\right)$ hexatriene & -0.00737 & 0.00181 & -3.9365 \\
$\operatorname{TS}\left(\mathrm{C}_{2 \mathrm{v}}\right)$ octatetraene & -0.00608 & -0.00054 & -4.3555 \\
\hline
\end{tabular}




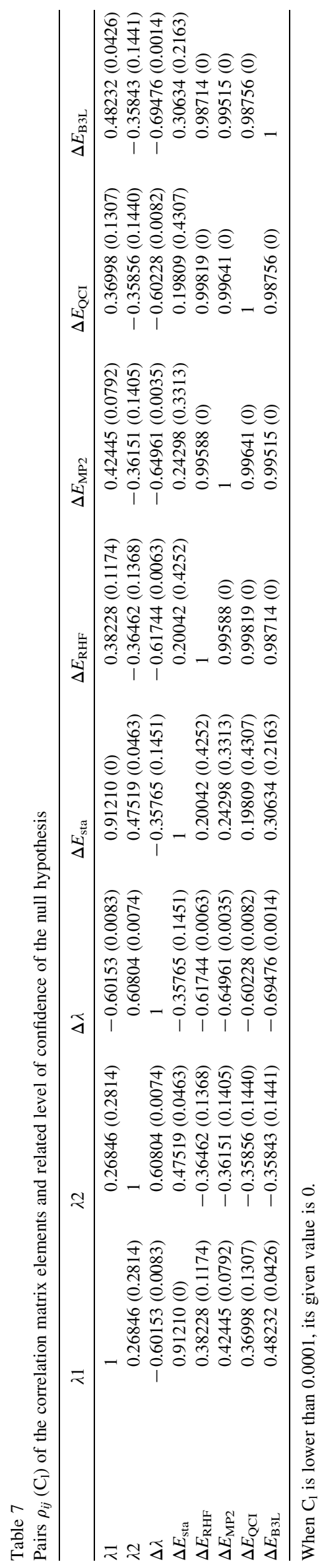

with the triplet HF instability was previously emphasized [42]: the larger the electronic correlation, the larger the HF instability, i.e. the smaller $\lambda 1$. Let us point out here that the existence of the electronic delocalization can be suggested on the basis of the geometry of the molecular system: it must present the possibility of conjugation of the unsaturated bonds, i.e., the system should be more or less planar, it should show high symmetry (more or less equal $\mathrm{C}-\mathrm{C}$ bond lengths) and $\mathrm{C}-\mathrm{C}$ distances should be of the order of $1.35-1.40 \AA$ or less. Nevertheless, the estimation of the importance of the delocalization is measured through the resonance energy, which can be defined in several ways [54].

\section{Butadiene, hexatriene, octatetraene}

\subsection{Molecular exploration}

The studied conformations of hexatriene and octatetraene are presented in Figs. 2 and 3. For butadiene, the situation is much simpler since only four conformers are considered: the planar cis (TS), the cis(min), the trans(min) and a so-called twis(TS) for which the dihedral $\mathrm{C}=\mathrm{C}-\mathrm{C}=\mathrm{C}$ is respectively equal to $0,40.31,180$ and $100.46^{\circ}$. The three or four lowest eigenvalues of $\mathscr{H}$ are presented in Tables $3-5$ as well as the stabilisation energy $\Delta \mathrm{E}_{\text {sta }}=\mathrm{E}(\mathrm{UHF})-\mathrm{E}(\mathrm{RHF})$.

For the three polyenes, there exists one conformation characterized by a quasi null $\lambda 1$ eigenvalue $(|\lambda 1|<0.005$ a.u.). At these geometries corresponding to dihedrals around the single bonds of about $100^{\circ}$, one, two or three other eigenvalues are very small $(\lambda i<0.015$ a.u. $)$ and the means of all these small $\lambda i$ lies around 0.007 a.u. This corresponds to situations where the $\pi$ clouds of the double bonds are hardly interacting, as if the polyenes were just a set of independent ethylenes for which $\lambda 1$ is around in fact 0.007 a.u. Once again, the conjugation pattern has a role to play. This value of $100^{\circ}$ for which the conjugation seems to be broken appears again for a second peculiar conformation of octatetraene, $\mathrm{TS}(\mathrm{C} 2)$, which is characterized by four smallest eigenvalues two by two degenerate, leading to a situation equivalent to a superposition of two butadienes: $\lambda 1 \approx \lambda 2 \approx-0.023$ a.u. and $\lambda 3 \approx \lambda 4 \approx 0.047$ a.u., i.e. the two lowest eigenvalues for the trans-butadiene (Fig. 4).

For the octatetraene, a second eigenvalue of $\mathcal{H}, \lambda 2$, appears to be negative, and generally speaking, the level of instability given by the absolute value of either $\lambda 1$ or $\Delta E_{\text {sta }}$ increases as the number of $\pi$ electrons increases. This has already been pointed out previously [42]. Since the energy is an extensive variable, i.e. depends on the number of particles in the system, and since it was clearly put in relation with the $\pi$ electronic system, it seems interesting to concentrate on the instabilityrelated quantities per $\pi$ electrons $\left(n_{\pi}\right)$, i.e. $\lambda i / n_{\pi}$ or $\Delta E_{\mathrm{sta}} / n_{\pi}$. Some of these quantities are presented in Table 6. Going from butadiene to octatetraene, $\lambda 2 / n_{\pi}$ and $\Delta E_{\text {sta }} / n_{\pi}$ decrease while $\lambda 1 / n_{\pi}$ shows a different behavior: it decreases from butadiene to hexatriene and then increases to about the value in butadiene. Nevertheless, the sum of $\lambda i / n_{\pi}$ always decreases. Moreover, when the conjugation is hindered (cis-butadiene and 
Table 8

Snedecor-Fisher $F$ value and associated significance probability $\operatorname{Pr}>$ F [52] for 18 molecular systems and 8 variables according to the ANOVA procedure

\begin{tabular}{lrr}
\hline & $F$ value & Pr $>$ F \\
\hline$\lambda 1$ & 2.06 & 0.1617 \\
$\lambda 2$ & 23.09 & $<0.0001$ \\
$\Delta \lambda$ & 1.00 & 0.3921 \\
$\Delta E_{\text {sta }}$ & 5.52 & 0.0160 \\
$\Delta E_{\mathrm{RHF}}$ & 1.04 & 0.3777 \\
$\Delta E_{\mathrm{MP} 2}$ & 1.00 & 0.3923 \\
$\Delta E_{\mathrm{QCI}}$ & 1.15 & 0.3426 \\
$\Delta E_{\mathrm{B} 3 \mathrm{~L}}$ & 1.00 & 0.3919 \\
\hline
\end{tabular}

$\min \left(\mathrm{C}_{2}\right)$ of hexatriene and octatetraene), $\lambda 1 / n_{\pi}$ increases, i.e. the instability decreases.

\subsection{Statistical analysis}

The variables considered in the statistical analysis are the following. The first lowest eigenvalues of $\mathscr{H} \lambda 1$ and $\lambda 2$ and their difference $\Delta \lambda$, the stabilisation energy $\Delta E_{\text {sta }}$, and the energies of the studied conformers at the RHF, MP2, QCISD and B3LYP levels $\Delta E_{\mathrm{RHF}}, \Delta E_{\mathrm{MP} 2}, \Delta E_{\mathrm{QCI}}, \Delta E_{\mathrm{B} 3 \mathrm{~L}}$. These latter energies are calculated by reference to the lowest energy conformer, i.e. the all-trans in each case. They were included in the analysis because it was previuosly pointed out that the electronic correlation has some role to play in the appearance of the instability. The correlation matrix elements as well as the confidence levels $C_{1}$ under the null hypothesis (Section 2) are given in Table 7.
It is obvious that the four energy variables are redundant since the $C_{1}$ values are 0 for all their related correlation elements. This is not surprising since the largest amount of all these energies comes from the 'not- $\pi$ ' electrons contribution which must vary in the same way at all the levels.

It also appears that $\Delta E_{\mathrm{sta}}$ is fully correlated with $\lambda 1$, and that $\Delta \lambda$ is redundant with both $\lambda 1$ and $\lambda 2$.

A canonical variance analysis based upon the a priori criterion of belonging to one of the unsaturated groups is performed with different sets of variables, differing by the number of energy variables $\Delta E_{X}$. For the initial eight variables, the equivalence between the four $\Delta E_{X}$ clearly appears as shown in Table 8. Moreover, the results with one or zero energy variables are very similar, as presented in Fig. 5. For the two variable sets chosen, only one bad sorting results from the analysis: $\mathrm{PC} 3\left(\mathrm{C}_{2}\right)$ of octatetraene is found with all the hexatriene conformers. From this analysis, the variable that can discriminate the groups is $\lambda 2$ (Table 8).

A further study of the influence of the electronic correlation energy was performed by considering two supplementary variables $\Delta E_{\text {corr }}(\mathrm{MP} 2)$ and $\Delta E_{\text {corr }}(\mathrm{QCI})$ calculated by reference to the electronic correlation energy for the trans conformers as $\mathrm{E}(\mathrm{X}$,conformer) $-\mathrm{E}$ (RHF,conformer $)-[\mathrm{E}(\mathrm{X}$, trans-conf $)-\mathrm{E}(\mathrm{RHF}$, trans-conf $)], \quad \mathrm{X}=\mathrm{MP} 2$ or QCISD. The correlation coefficients of these two variables with the previous ones within the a priori defined groups are presented in Table 9. It shows up that $\Delta E_{\text {corr }}(\mathrm{QCI})$ highly correlates with all the energy variables, which is expected. As a matter of fact, the larger the number of electrons (and thus the higher the electronic correlation
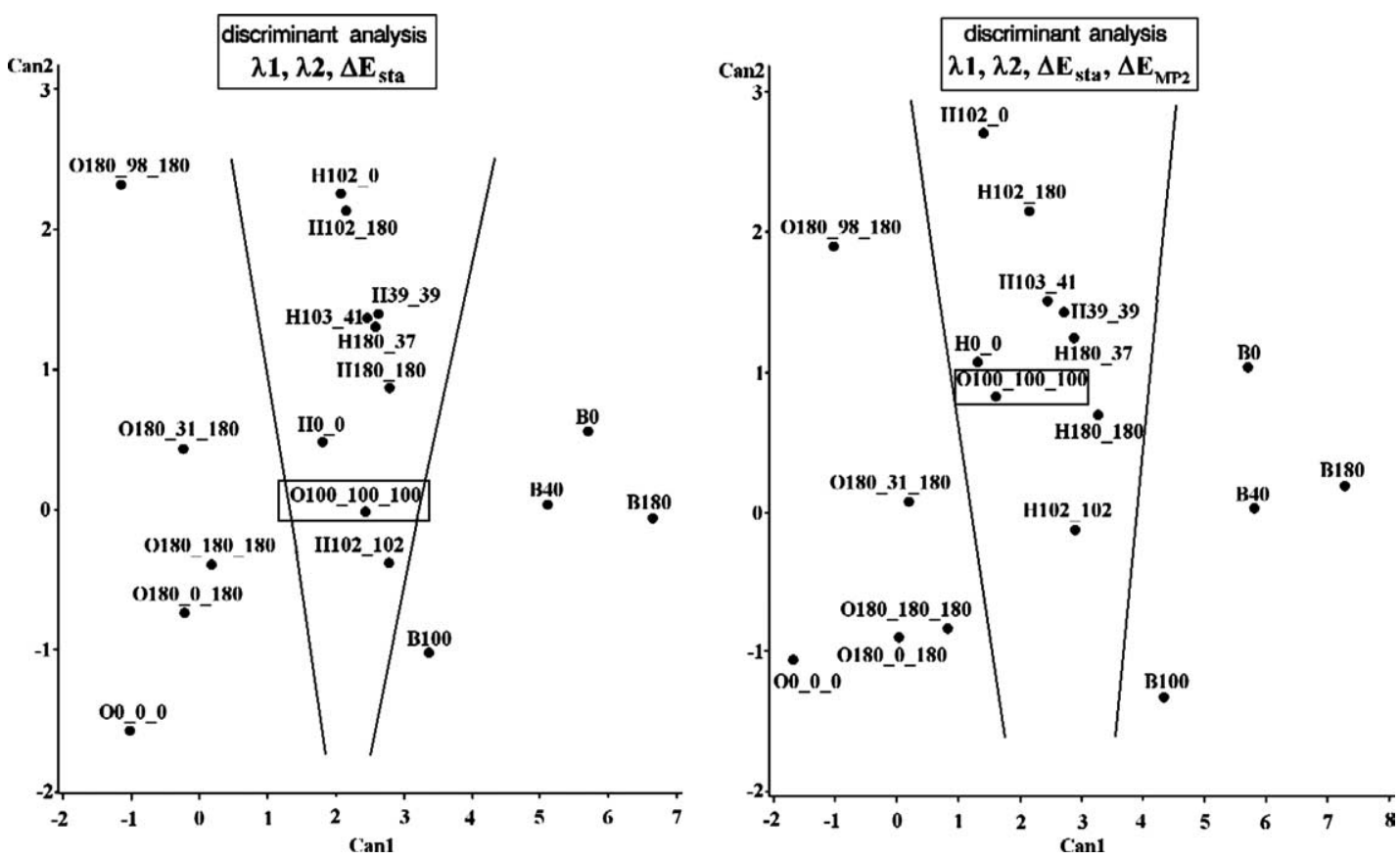

Fig. 5. Result of the discriminant analysis for the 18 studied conformers of butadiene, hexatriene and octatetraene, considering two variable sets, either $\{\lambda 1, \lambda 2$ and $\left.\Delta E_{\text {sta }}\right\}$ or $\left\{\lambda 1, \lambda 2, \Delta E_{\text {sta }}\right.$ and $\left.\Delta E_{\mathrm{MP} 2}\right\}$. The points of the graph are labelled by the initial of the unsaturated compound followed by the value(s) of the dihedrals in the corresponding conformer. 


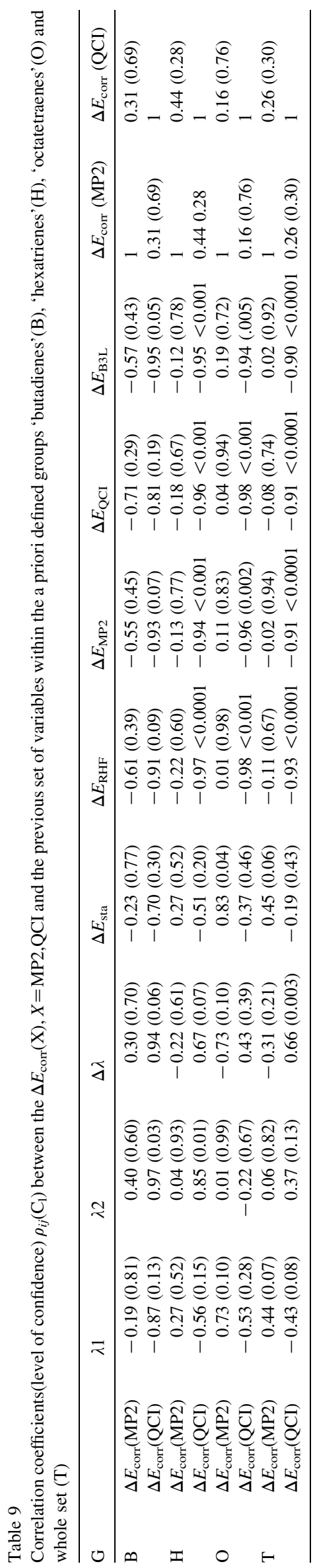

energy), the higher the total energy. However, this is not observed for the $\Delta E_{\text {corr }}(\mathrm{MP} 2)$ probably because the correlation energy calculated at the MP2 level is not good enough. Apparently, for the smaller systems (butadienes and hexatrienes), there is a certain degree of relationship between $\Delta E_{\text {corr }}(\mathrm{QCI})$ and $\lambda 2$ but this disappears for octatetraene. However, there is no correlation between the energy of electronic correlation and the quantities related to the instability, i.e. $\lambda 1$ or $\Delta E_{\text {sta. }}$ A canonical variance analysis with the variables $\lambda 1, \Delta \lambda, \Delta E_{\text {sta }}$ and $\Delta E_{\text {corr }}(\mathrm{QCI})$ produces very similar results as previously since only one bad sorting is obtained: $\mathrm{PC} 3\left(\mathrm{C}_{2}\right)$ of octatetraene again found with the hexatrienes. It thus appears that the concept of electronic correlation linked with the HF instability [42] is vague. In solid state physics, it is well admitted that the observed Peierls instabilities are due to electronic correlation [55-59] and in the cases treated here, it should be interesting to determine the electronic correlation energy in the $\pi$ electronic cloud only.

\section{Conclusions}

From the study of $\mathrm{NH}_{2}$ and $\mathrm{NO}_{\mathrm{x}}$ substituted ethylenes, it appears that conjugation and mesomeric effects play an important role in the HF instability of unsaturated compounds. The donating character of the amino substituent(s) clearly results in a more delocalized wave function for the $\pi$ electrons, which should be related with a smaller electronic correlation within the $\pi$ cloud.

This is confirmed by the results obtained on small polyenes in different conformations. It was found that for the dihedral values, around $100^{\circ}$, where the conjugation pattern is broken, the instability nearly disappears probably due to a decrease in electronic correlation.

It was also shown that the intrinsic instability, represented by either $(\lambda 1+\lambda 2) / n_{\pi}$ or $\Delta E_{\mathrm{sta}} / n_{\pi}$, increases with the number of $\pi$ electrons $n_{\pi}$.

A statistical analysis on the compounds allowed to reveal the role of $\lambda 2$ in discriminating between the groups. It also underscored the correlation between $\lambda 1$ and $\Delta E_{\text {sta }}$, as well as the complete equivalence of all the total energy variables. Moreover, no relation between the instability variables and the correlation energy could be brought to the fore, probably because the largest amount of this energy is not due to the $\pi$ electrons.

A further study on the HF instabilities should deal with the whole spectrum of $\lambda i$ eigenvalues of $\mathscr{H}$ instead of limiting oneself to $\lambda 1$ and $\lambda 2$. Since the triplet HF instability is a characteristic of unsaturated conjugated compounds, and is thus related with the delocalized $\pi$ electrons, it is expected that it could appear in cases where valence electrons shared by several atomic centers are rather loosely bound to the skeleton. This leads to two possibilities: either the volatile electrons participate in a delocalized wave function, in which case a RHF description is adequate, or they present a degree of localization around certain atomic centers thus rendering the RHF framework less adapted. This very localization makes the problem linked with the distances between atomic centers or 
with the question of the attraction basin for each electron: is the nuclear skeleton sufficiently compact for all the loosely bound electrons to see it as a free playground or are the atoms sufficiently distant from one another for preventing the electrons to move freely around the whole skeleton? There is no answer before any calculation is done and the analysis of the electronic properties of such systems should be performed with several tools. One should differentiate the two problems of determining some physical observables at a quantitative level with a good confidence on one hand, and of studying qualitatively a property of the system on the other hand.

In the first case, a high-quality calculation level is required and in anyway a correlated multideterminantal wave function is required. Thus working with a RHF zero order wave function remains a good starting point and the existence of a triplet HF instability is not important. The geometry optimization should be conducted with a multideterminantal wave function which allows by construction delocalized as well as localized electronic configurations but this is not possible for large systems. Apparently, the density functional theory (DFT)[60] leads to instabilities for larger interatomic distances [61,62] and could be used for geometry optimizations of large systems with good confidence. As to the electronic properties, a multideterminantal wave function is necessary, be it of the CI or MCSCF type. Moreover, the valence bond methods are certainly very powerful tools to deal with the problem of electronic conjugation $[63,64]$.

In the second case, the level at which the property will be evaluated will of course depend on the problem. For instance, the qualitative comparison of proton affinities in a series of molecules could be performed at the HF and MP2 levels; this will be sufficient to reproduce the general trend and to compare the global effect of the electronic correlation.

One point that should be emphasized here is the following. If a molecule is subject to a triplet HF instability, its geometry should not be optimized with a relaxed UHF wave function because this latter does no longer possess the symmetry of the molecule. Similarly, a frequency determination should not be done with a relaxed UHF wave function for the same reason. Thus, the triplet HF instability does reveal an electronic peculiar behavior due to some kind of electronic correlation but the properties should still be calculated with a RHF-based wave function since it keeps the symmetry properties of the nuclear skeleton.

\section{Uncited references}

[60]. [61]. [62]. [63]. [64].

\section{Acknowledgements}

This work was supported by the Belgian program on Interuniversity Poles of Attraction initiated by the Belgian State, Prime Minister's Office, Services fédéraux des affaires scientifiques, techniques et culturelles (PAI no P5/33). GD is chercheur qualifié of the FNRS, Brussels. CG is grateful to the FRIA for a grant.

\section{References}

[1] D.R. Hartree, Proc. Camb. Philos. Soc. Math. Phys. Sci. 24 (1928) 89.

[2] J.C. Slater, Phys. Rev. 34 (1929) 1293.

[3] J.C. Slater, Phys. Rev. 35 (1930) 210.

[4] C. Cohen-Tannoudji, B. Diu, F. Laloë, Mécanique quantique, Hermann, Paris, France, 1973.

[5] F. Jensen, Introduction to Computational Chemistry, Wiley, New York, 1999.

[6] A. Szabo, N.S. Ostlund, Modern Quantum Chemistry. Introduction to Advanced Electronic Structure Theory, Dover, New York, 1996.

[7] W. Pauli, Z. Phys. 31 (1925) 765.

[8] W. Pauli, Phys. Rev. 58 (1940) 716

[9] H. Margenau, G.M. Murphy, The Mathematics of Physics and Chemistry, vol. I, Van Nostrand, Princeton, NJ, 1956.

[10] V.A. Fock, Z. Phys. 61 (1930) 126.

[11] F.A. Cotton, Chemical Applications of Group Theory, Wiley-Interscience, New York, 1971.

[12] J.C. Slater, Phys. Rev. 35 (1930) 509.

[13] C.A. Coulson, I. Fisher, Philos. Mag. 40 (1949) 386.

[14] D.J. Thouless, The Quantum Mechanics of Many-Body Systems, Academic Press, New York, 1961.

[15] W.H. Adams, Phys. Rev. 127 (1962) 1650.

[16] A.W. Overhauser, Phys. Rev. 128 (1962) 1347.

[17] P.O. Löwdin, Rev. Mod. Phys. 35 (1963) 496.

[18] J. Koutecky, J. Chem. Phys. 46 (1967) 2443.

[19] J. Cisek, J. Paldus, J. Chem. Phys. 47 (1967) 3976.

[20] R. Lefebvre, Y.G. Smeyers, Int. J. Quantum Chem. 1 (1967) 403.

[21] R.E. Stanton, J. Chem. Phys. 48 (1968) 257.

[22] H. Fukutome, Prog. Theor. Phys. 40 (1968) 998.

[23] H. Fukutome, Prog. Theor. Phys. 40 (1968) 1227.

[24] P.O. Löwdin, Adv. Chem. Phys. 14 (1969) 283.

[25] J. Paldus, J. Cizek, Chem. Phys. Lett. 3 (1969) 1.

[26] A. Hilbert, C.A. Coulson, J. Phys. B 2 (1969) 458.

[27] J. Paldus, J. Cizek, J. Polym. Sci. 29 (1970) 199.

[28] J. Paldus, J. Cizek, Phys. Rev. A 2 (1970) 2268.

[29] J. Paldus, J. Cisek, J. Chem. Phys. 52 (1970) 2919.

[30] J. Paldus, J. Cizek, J. Chem. Phys. 54 (1971) 2293.

[31] N.S. Ostlund, J. Chem. Phys. 57 (1972) 2994.

[32] H. Fukutome, Prog. Theor. Phys. 47 (1972) 1156.

[33] W.G. Laidlaw, Int. J. Quantum Chem. 7 (1973) 87.

[34] H. Fukutome, Prog. Theor. Phys. 49 (1973) 22.

[35] K. Yamaguchi, T. Fueno, H. Fukutome, Chem. Phys. Lett. 22 (1973) 461.

[36] H. Fukutome, Prog. Theor. Phys. 52 (1974) 115.

[37] H. Fukutome, Prog. Theor. Phys. 52 (1974) 1766.

[38] R. Seeger, J.A. Pople, J. Chem. Phys. 66 (1977) 3045.

[39] G. Chambaud, B. Levy, P. Millie, Theor. Chim. Acta 48 (1978) 103.

[40] H. Fukutome, Int. J. Quantum Chem. 20 (1981) 955.

[41] N. Vancampenhout, G. Dive, D. Dehareng, Int. J. Quantum Chem. 60 (1996) 911.

[42] D. Dehareng, G. Dive, J. Comput. Chem. 21 (2000) 483.

[43] D. Dehareng, G. Dive, A. Moradpour, Int. J. Quantum Chem. 76 (2000) 552 .

[44] B. Braïda, D. Lauvergnat, P.C. Hiberty, J. Chem. Phys. 115 (2001) 90.

[45] D. Dehareng, G. Dive, C. Geron, Recent Research Developments in Chemical Physics, vol. 3, Transworld Research Network, Trivandrum, 2002 (Part 1) p. 193.

[46] M.J. Frisch, G.W. Trucks, H.B. Schlegel, G.E. Scuseria, M.A. Robb, J.R. Cheeseman, V.G. Zakrzewski, J.A. Montgomery Jr., R.E. Stratmann, J.C. Burant, S. Dapprich, J.M. Millam, A.D. Daniels, K.N. Kudin, M.C. Strain, O. Farkas, J. Tomasi, V. Barone, M. Cossi, R. Cammi, B. Mennucci, C. Pomelli, C. Adamo, S. Clifford, J. Ochterski, G.A. Petersson, P.Y. Ayala, Q. Cui, K. Morokuma, D.K. Malick, A.D. Rabuck, K. Raghavachari, J.B. Foresman, J. Cioslowski, J.V. Ortiz, A.G. Baboul, B.B. Stefanov, G. Liu, A. Liashenko, P. 
Piskorz, I. Komaromi, R. Gomperts, R.L. Martin, D.J. Fox, T. Keith, M.A. Al-Laham, C.Y. Peng, A. Nanayakkara, C. Gonzalez, M. Challacombe, P.M.W. Gill, B. Johnson, W. Chen, M.W. Wong, J.L. Andres, C. Gonzalez, M. Head-Gordon, E.S. Replogle, J.A. Pople, Gaussian 98, Revision A.7, Gaussian Inc., Pittsburgh, PA, 1998.

[47] A.D. McLean, G.S. Chandler, J. Chem. Phys. 72 (1980) 5639.

[48] R. Krishnan, J.S. Binkley, R. Seeger, J.A. Pople, J. Chem. Phys. 72 (1980) 650.

[49] M. Head-Gordon, J.A. Pople, M.J. Frisch, Chem. Phys. Lett. 153 (1988) 503.

[50] J.A. Pople, M. Head-Gordon, K. Raghavachari, J. Chem. Phys. 87 (1987) 5968.

[51] A.D. Becke, J. Chem. Phys. 98 (1993) 5648.

[52] D.F. Morrisson, Multivariate Statistical Methods, Duxbury Advanced Series, Duxbury Press, North Scituate, MA, 2002.

[53] SAS Institute Inc., Cary, NC, USA.

[54] M. Bühl, A. Hirsch, Chem. Rev. 101 (2001) 1153.
[55] R.E. Peierls, Quantum Theory of Solids, Oxford University Press, Oxford, 1955.

[56] G. Beni, P. Pincus, J. Chem. Phys. 57 (1972) 3531.

[57] V.A. Kuprievich, Phys. Rev. B 50 (1994) 16872.

[58] S.-L. Drechsler, J. Málek, G. Paasch, K. Hallberg, Synth. Met. 101 (1999) 386.

[59] Q. Yuan, T. Nummer, T. Kopp, Eur. Phys. J. B 22 (2001) 37.

[60] R.G. Parr, W. Yang, Density Functional Theory of Atoms and Molecules, Oxford University Press, New York, 1989.

[61] R. Bauernschmidtt, R. Ahlrichs, J. Chem. Phys. 104 (1996) 9047.

[62] M.O. Jensen, T. Thorsteinsson, A.E. Hansen, Int. J. Quantum Chem. 90 (2002) 616.

[63] D.L. Cooper, S.C. Wright, J. Gerratt, M. Raimondi, J. Chem. Soc., Perkin Trans. II (1989) 255.

[64] D.L. Cooper, S.C. Wright, J. Gerratt, M. Raimondi, J. Chem. Soc., Perkin Trans. II (1989) 255; D.L. Cooper, S.C. Wright, J. Gerratt, M. Raimondi, J. Chem. Soc., Perkin Trans. II (1989) 263. 